\title{
End-to-end simulations of the E-ELT/METIS coronagraphs
}

\author{
Brunella Carlomagno $^{a}$, Olivier Absil ${ }^{a}$, Matthew Kenworthy ${ }^{c}$, Garreth Ruane $^{d}$, \\ Christoph U. Keller ${ }^{c}$, Gilles Otten ${ }^{c}$, Markus Feldt ${ }^{e}$, Stefan Hippler ${ }^{e}$, Elsa Huby ${ }^{a}$, \\ Dimitri Mawet $^{d}$, Christian Delacroix ${ }^{f}$, Jean Surdej ${ }^{a}$, Serge Habraken ${ }^{a}$, Pontus Forsberg ${ }^{b}$, \\ Mikael Karlsson ${ }^{b}$, Ernesto Vargas Catalan ${ }^{b}$ and Bernhard R. Brandl ${ }^{c}$ \\ ${ }^{a}$ Space sciences, Technologies, and Astrophysics Research (STAR) Institute, Université de \\ Liège, 19C Allée du Six Août, B-4000 Sart Tilman, Belgium; \\ ${ }^{b}$ Ångström Laboratory, Uppsala University, Lägerhyddsvägen 1, SE-751 21 Uppsala, Sweden; \\ ${ }^{c}$ Leiden Observatory, Leiden University, P.O. Box 9513, 2300 RA Leiden, The Netherlands; \\ ${ }^{d}$ California Institute of Technology, 1200 E. California Blvd., Pasadena, CA 91125, USA; \\ e Max-Planck-Institut für Astronomie, Königstuhl 17, 69117 Heidelberg, Germany; \\ ${ }^{f}$ Sibley School of Mechanical and Aerospace Engineering, Cornell University, Ithaca, NY \\ 14853, USA;
}

\begin{abstract}
The direct detection of low-mass planets in the habitable zone of nearby stars is an important science case for future E-ELT instruments such as the mid-infrared imager and spectrograph METIS, which features vortex phase masks and apodizing phase plates (APP) in its baseline design. In this work, we present end-to-end performance simulations, using Fourier propagation, of several METIS coronagraphic modes, including focal-plane vortex phase masks and pupil-plane apodizing phase plates, for the centrally obscured, segmented E-ELT pupil. The atmosphere and the $\mathrm{AO}$ contributions are taken into account. Hybrid coronagraphs combining the advantages of vortex phase masks and APPs are considered to improve the METIS coronagraphic performance.
\end{abstract}

Keywords: Exoplanets detection, Mid-IR, Vector Vortex Coronagraphs, Apodizing Phase Plate coronagraphs, E-ELT/METIS instrument, end-to-end simulations

\section{INTRODUCTION}

The direct detection of exoplanets is one of the most important science cases for the future extremely large telescopes (ELTs). By analyzing the planet spectrum, the atmospheric composition can be retrieved and eventually biosignatures can be detected. Direct imaging, however, presents some challenges: the small angular separation between the star and its planet and the large contrast between the two bodies. Observing in the mid-infrared regime $(3-13 \mu \mathrm{m})$ can attenuate the contrast difference (for an Earth-like planet, the contrast eases from $10^{-10}$ in the visible to $10^{-7}$ in the infrared; for a Jupiter-like planet, from $10^{-8}$ to $10^{-7}$ ). A coronagraphic technique can also be implemented to suppress the diffracted light from the star and permit the light from the companion to pass. Coronagraphs can be grouped into four categories, depending on the position of the mask (focus or pupil) and the occultation method (amplitude or phase).

- The pupil masks redistribute the light in the image plane, and they are generally one-stage system, with an apodizer in the optical layout. The Apodizing Phase Plate ${ }^{1}$ is a pupil-plane phase coronagraph, consisting in a phase mask placed at the pupil plane. It is relatively insensitive to residual tip/tilt in the focal plane, which makes it a very robust coronagraph. It has been installed and tested on several near-infrared camera installed on large telescopes: VLT/NACO,${ }^{2,3} \mathrm{LBT} / \mathrm{LMIR}$ cam, ${ }^{4}$ Magellan/CLIO (Otten et al., in prep). The original version of the $\mathrm{APP}^{1}$ used variations in the thickness $d$ of a Zinc Selenide glass to impart a position dependent variation in optical path length, and therefore a variation in phase at a central wavelength $\lambda_{0}$ through $\Delta \phi=2 \pi d(n-1) / \lambda_{0}$. This implementation of APP led to the imaging of $\beta$ Pic $\mathrm{b}^{3}$

Further author information: brunella.carlomagno@ulg.ac.be; phone: +32 43669739; ago.ulg.ac.be 
and the discovery of HD 100546b. ${ }^{5}$ More recently, the variation of phase has been implemented as an orientation of the fast axis of a birefringent material, ${ }^{6}$ which has enabled the optic to be made broadband up to an octave in wavelength in the laboratory ${ }^{7}$ and is now demonstrated in on-sky observations. ${ }^{8}$ This new version is called vector APP (vAPP).

- The focal-plane masks have at least two stages of filtering, one at the focal plane and a second at the second pupil plane. The vector vortex phase mask is a focal-plane phase coronagraph. It generates a phase shift (see Fig.1, right) that deflects the light from the star beyond the area of interest. This light is then blocked with an opaque diaphram, the Lyot Stop, placed at the second pupil plane. The effect of this vector vortex is to make the incoming horizontal polarization turn twice as fast as the azimuthal coordinate for an on-axis source. When the vector vortex has completed the rotation with a phase ramp of $2 * 2 \pi$, the produced optical vortex is of charge 2 (see Fig.1, left). The AGPM (Annular Groove Phase Mask) ${ }^{9,10}$ is a charge-2 vector vortex coronagraph, obtained with rotationally symmetric subwavelength gratings etched into diamond substrates. ${ }^{11,12}$ Since its first light in the VLT/NACO ${ }^{13}$ instrument in late 2012 , its results have been remarkable, with a series of new instruments in its portfolio (LBT/LMIRCam, Keck/NIRC2, VLT/VISIR). Its impressive characteristics (small inner working angle, high throughput, clear off-axis $360^{\circ}$ discovery space, achromaticity over $20 \%$ bandwidth, simplicity) make it an attractive solution for future E-ELT instruments, and METIS ${ }^{14}$ in particular.

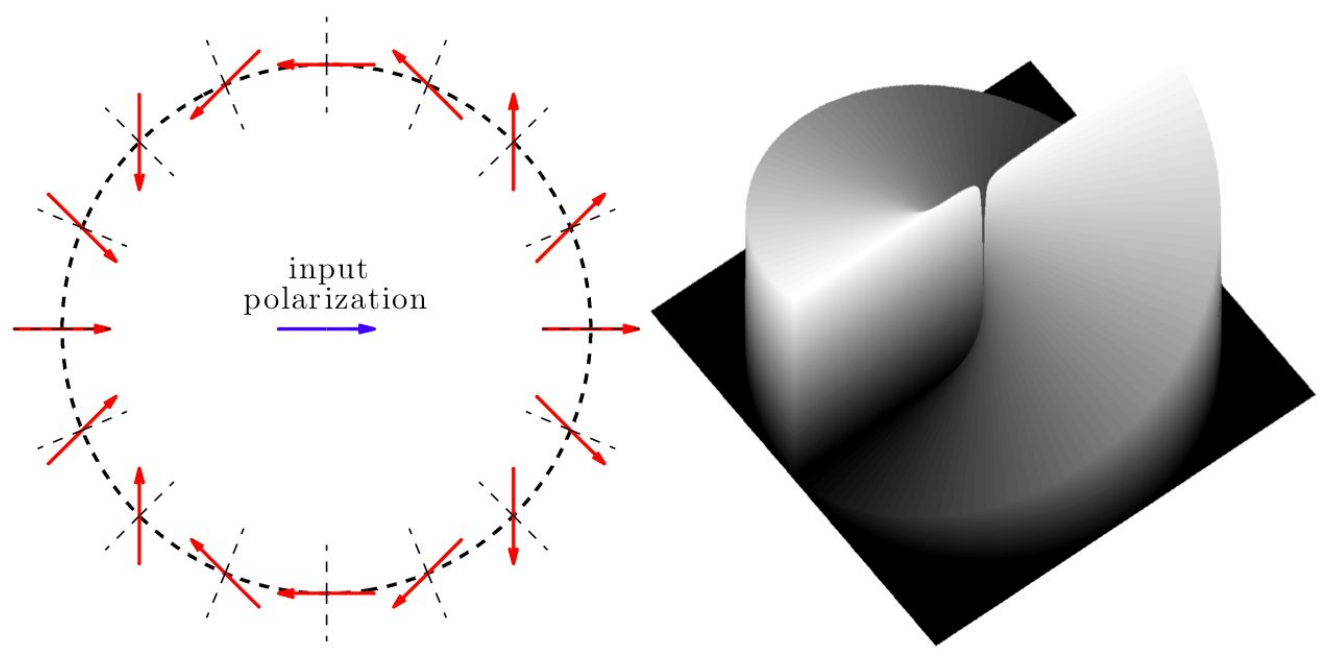

Figure 1: Left: Illustration of the spatial variation of the orientation of the fast axis of an HWP. Right: Computed $2 \times 2 \pi$ phase ramp created by a charge- 2 vortex.

\section{THE E-ELT/METIS INSTRUMENT}

The mid-infrared imager and spectrograph METIS ${ }^{15}$ is one of the first-light instruments for the European ELT. Its main science cases include exoplanet detection, with the aim of studying the physical and chemical properties of the companions, and the formation and evolution of protoplanetary disks, for a better understanding of the planetary diversity. Its baseline design features vortex phase masks and apodizing phase plates $\left(\mathrm{APP}^{1}\right)$ for imaging and spectroscopy at $\mathrm{L}, \mathrm{M}$ and $\mathrm{N}$ atmospheric bands. ${ }^{16}$

The E-ELT pupil contains 798 quasi-hexagonal segments, $1.45 \mathrm{~m}$ from side to side, divided into 6 sectors (see Fig.2, left), with six 50cm-wide spider arms. It features a large central obstruction (around $11 \mathrm{~m}$ in diameter, $30 \%$ of the outer diameter), which is not ideal for coronagraphic applications. Natural guide star single-conjugated adaptive optics simulations under YAO have been used to estimate the residual turbulence at the entrance of the METIS instrument. These simulations assume 74 sub-apertures across the pupil, sampled with $6 \times 6$ pixels each, with $0.5 \mathrm{~m}$ sub-aperture size, and 3992 actuators over the deformable mirror. The assumed conditions are very good: $0.44 "$ zenith seeing at $3 \mu \mathrm{m}, \mathrm{K}$-star of $4.45 \mathrm{mag}, 30^{\circ}$ away from zenith. The simulations include the 


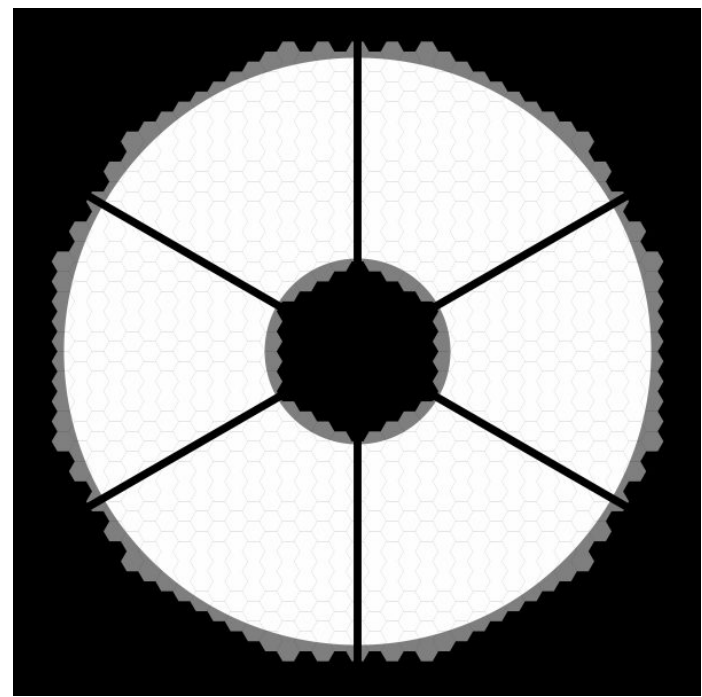

(a)

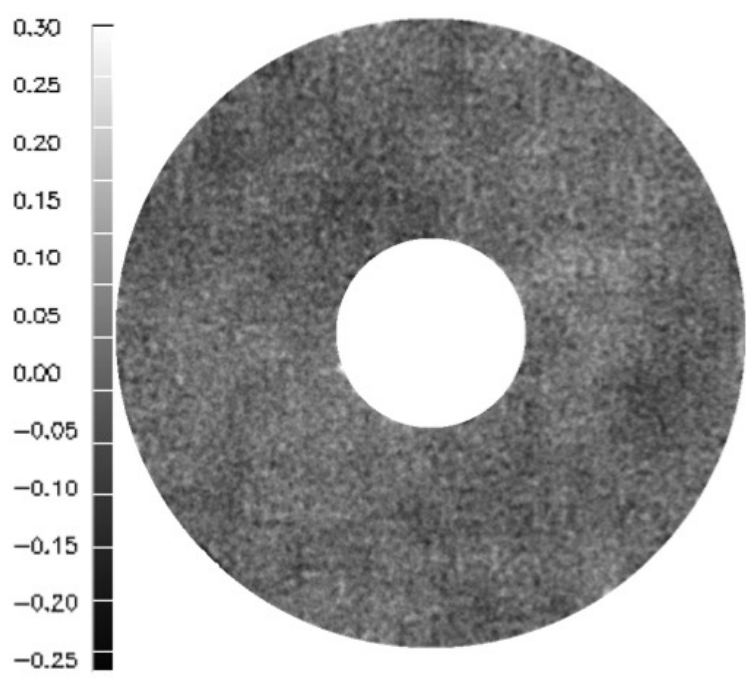

(b)

Figure 2: Left: Pupil of the segmented E-ELT, and the inscribed circular pupil considered in this work. Right: Illustration of one residual turbulent phase screen (in $\mu m$ ) after correction by the METIS SCAO ${ }^{17,18}$

atmospheric contribution to tip-tilt jitter, but do not include the effect of telescope vibration, due e.g. to wind shake. The wavefront sensor is a Shack-Hartmann, operating at K band. In the METIS preliminary design, the cold stop, placed upstream the coronagraph and the WFS, had a circular shape, therefore the E-ELT pupil has also been circularized (see Fig.2, left) for our coronagraphic simulations: the outer diameter has been reduced to $37 \mathrm{~m}$, and the inner diameter set to $11.7 \mathrm{~m}$. This design might evolve into a dodecagonal configuration in the future to maximize throughput, but this wouldn't significantly change the conclusions of this study.

\section{SIMULATION}

The end-to-end simulations have been performed with the PROPER ${ }^{19}$ library, in the IDL environment. This library contains routines of optical propagation in the near-field and far-field conditions, using Fourier transform algorithms (angular spectrum, Fresnel approximation). The propagating wavefront is described by its amplitude and phase. In particular, the surface of constant phase is a plane for a perfectly collimated beam, measured perpendicularly to the optical axis. When the beam passes through a lens, the wavefront acquires a radius of curvature: the surface of constant phase is now a curve. The curvature can create a problem in the representation of the phase in a planar sampled grid, because at a certain distance from the propagation axis, the actual phase change between samples is greater than $2 \pi$, aliasing it. The implemented solution is to consider the phase as a phase error relative to a sphere with the known radius of curvature.

The distance from the beam waist delimiting the near-field and the far-field is defined by the Rayleigh distance. In the near-field, the best wavefront reference surface is planar, while in the far-field a curved surface is necessary. In the near-field, PROPER uses the angular spectrum algorithm for the propagation; in the far-field, it first propagates to the beam waist, and then to the new far-field point; between the two, it uses the Fresnel method. The simulations are subject to several assumptions: the system is on a straight line, implying a forward propagation for a positive distance, a mirror does not modify the direction of the beam nor the sign of the phase, but it will change the phase of the beam, and an aberration map with positive value will create a positive difference with respect to the default phase.

The implemented propagation layout is very simple (see Fig. 3 ): two pupil planes $-P P 1$ and $P P 2$ - and two focal planes $-F P 1$ and $F P 2$. The E-ELT/METIS pupil lies on the first pupil plane $P P 1$, while the detector occupies the second focal plane $F P 2$. The F-number is considered constant at 17.8. The simulations use a 


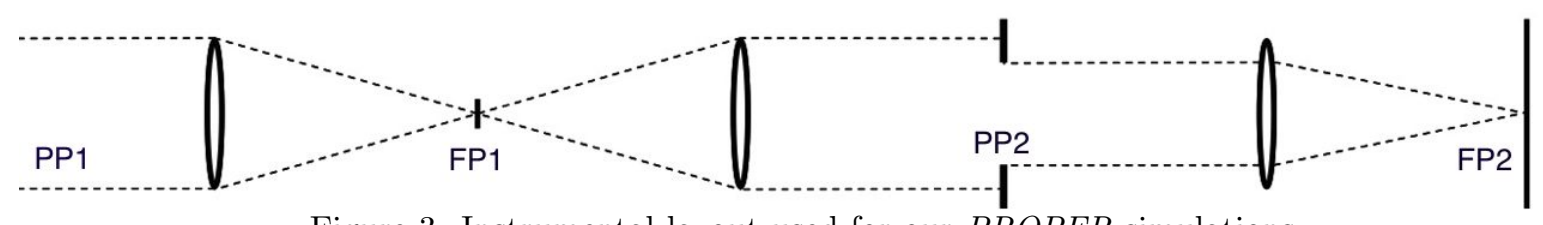

Figure 3: Instrumental layout used for our PROPER simulations.

2048 grid with a beam ratio of $0.25^{*}$, which entails a pixel value of $5.25 \mathrm{mas} / \mathrm{pix}$ in the L-band grid (where the simulation wavelength is set to $3.77 \mu \mathrm{m})$.

\subsection{The charge 2 Vortex Coronagraph (VC2)}

In this first coronagraphic configuration, the vector vortex phase mask is placed at the first focal plane $F P 1$, and a Lyot Stop at the second pupil plane PP2 (see Fig. 4(a)). In this preliminary study, the Lyot stop has not been optimized: the outer and inner radii and the spider thickness have simply been reduced or increased by a factor of $1 \%$. In this way, the surface area is reduced to $97 \%$ of the circularized E-ELT pupil. In this simulation, only the charge-2 vortex is taken into account. Figure 4(b) shows the final PSF. Instead of the perfect cancellation that a circular pupil would have provided, the non-circular obstructed pupil strongly reduces the contrast performance of the vortex mask. The presence of a central obstruction causes residual light to appear inside the diaphram, because of the intrinsic property of vortex coronagraphs of moving light in and out of circular apertures.

\subsection{The charge-2 Vortex Coronagraph with a Lyot Phase Mask (VC2 + LPM)}

The Lyot Phase Mask (LPM) ${ }^{20,21}$ is a phase-only (see Fig. 4(c)) optical element, placed at the Lyot stop PP2, that can improve the performance of the vortex coronagraph in a defined area of the focal plane (here, 3 to $20 \lambda / D$, see Fig. 4(d)). It is then a two-stage optical system. In practice, the vortex mask suppresses the light of the star, followed by the LPM creating a dark hole in the residual starlight, increasing the contrast locally. In this case, a full annulus dark hole is created (see Fig. 4(d)). It is very important to find a good trade-off between the desired level of contrast and the final throughput of the system: the darker the hole, the lower the final throughput (see section 4). At each iteration, the LPM phase is computed by a back and forward simulation between the Lyot stop plane and the image plane, where the dark hole is imposed. In this case, a 100 iterations LPM has been integrated into the simulations, leading to a final throughput of $20 \%$ (see table 1 ).

\subsection{The charge-2 Ring-Apodized Vortex Coronagraph (RAVC2)}

The RAVC2 $2^{22}$ combines a vortex coronagraph (focal plane $F P 1$ and pupil plane $P P 2$ ) with an amplitude ring apodizer in the first pupil plane $P P 1$. It is thus a three-stage optical system. The aim of the apodizer is to compensate for the presence of a large central obstruction in the instrument pupil by modulating the entrance pupil using a ring geometry (with chosen dimensions and transmittances) to perfectly cancel the on-axis source at the Lyot stop level.

Adding a pupil ring apodizer, with radius $r_{1}$ and amplitude transmission coefficient $t_{1}$ for $r_{1}<r<R$ and $t_{0}=1$ for $r_{0}<r<r_{1}$, the pupil field after the vortex mask and the Lyot Stop becomes:

$$
E_{L}(r)= \begin{cases}0 & r<r_{0} \\ -\left(\frac{r_{0}}{r}\right)^{2} & r_{0}<r<r_{1} \\ \left(1-t_{1}\right)\left(\frac{r_{1}}{r}\right)^{2}-\left(\frac{r_{0}}{r}\right)^{2} & r_{1}<r<R\end{cases}
$$

It is then possible to completely cancel the light within $r_{1}<r<R$ by varying the apodizer parameters, $t_{1}$ and $r_{1}$, which leads to an expression for the amplitude transmission parameter:

$$
t_{1}=1-\left(\frac{r_{0}}{r_{1}}\right)^{2}
$$

${ }^{*}$ The beam ratio is a variable in the PROPER environment, defined as the ratio between the pupil size and the simulation grid: 0.25 implies a pupil of 512 pizels in a 2048 simulation grid. 


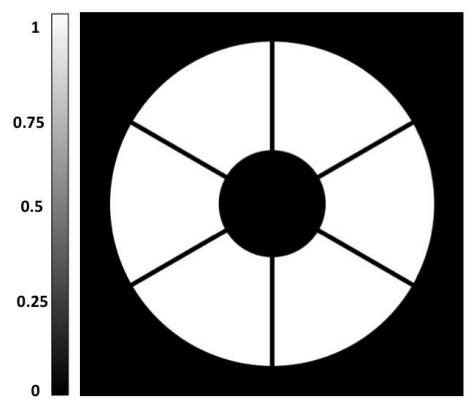

(a) Lyot Stop

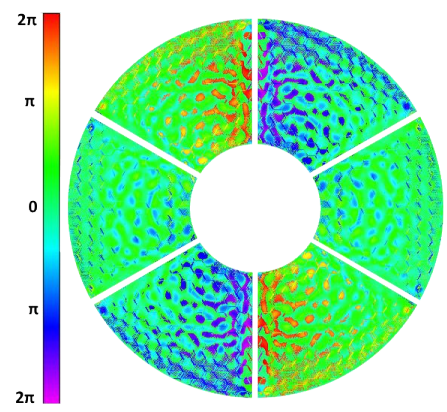

(c) LPM phase

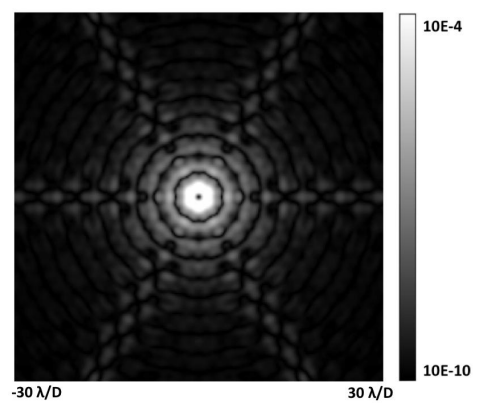

(b) VC2 psf

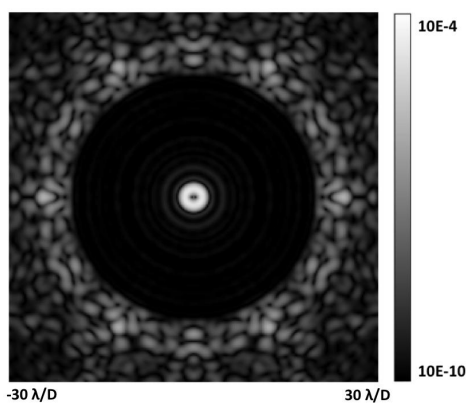

(d) $\mathrm{VC} 2+$ LPM psf

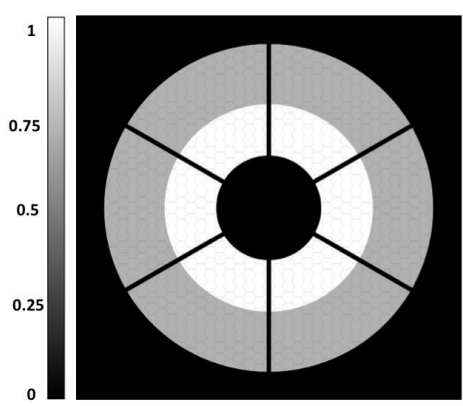

(e) RAVC2 pupil

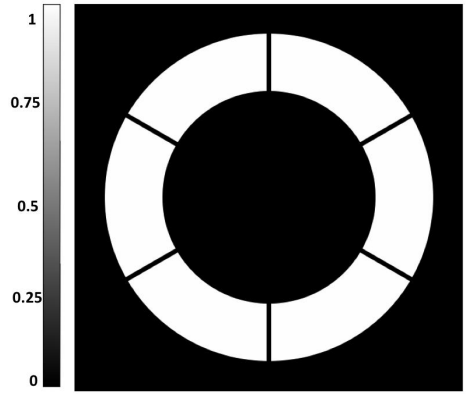

(f) RAVC2 Lyot stop

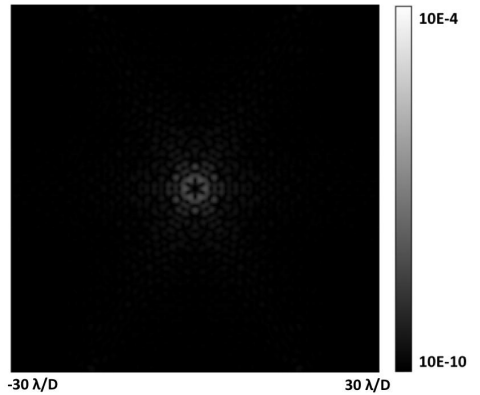

(g) RAVC2 psf

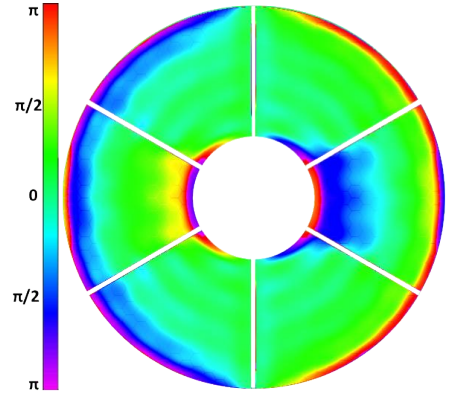

(h) APP phase

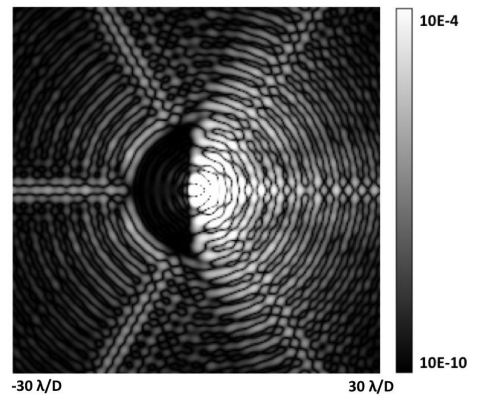

(i) APP psf

Figure 4: Input pupil, Lyot stop, phase and final psf (normalized to a total intensity of 1 in the non-coronagraphic psf) for the four coronagraphic concepts considered in this study. From top to bottom: VC2, VC2+LPM, RAVC2, APP. 
The second parameter, the radius $r_{1}$, is optimized by maximizing the throughput $\mathrm{T}$, defined as the ratio between the energy going through the ring and the energy normally transmitted by the centrally obscured telescope aperture:

$$
T=\frac{t_{1}^{2}\left(1-\left(\frac{r_{1}}{R}\right)^{2}\right)}{1-\left(\frac{r_{0}}{R}\right)^{2}}
$$

The final optimal parameters are defined as:

$$
\left\{\begin{array}{l}
t_{1, o p t}=1-\frac{1}{4}\left(R_{0}^{2}+R_{0} \sqrt{R_{0}^{2}+8}\right) \\
R_{1, o p t}=\frac{R_{0}}{\sqrt{1-t 1, o p t}}
\end{array}\right.
$$

where $R_{0}=r_{0} / R$ and $R_{1}=r_{1} / R$ are the relative radii. Note that the RAVC2 configuration depends only on the central obstruction radius: the larger its radius, the thinner the apodizer and the lower its transmission. For the circularized E-ELT/METIS pupil, we obtain (see Fig. 4(e)):

$$
R_{0}=0.3164 \rightarrow\left\{\begin{array}{l}
t_{1, o p t}=0.7498 \\
R_{1, o p t}=0.6326
\end{array}\right.
$$

Figure 4(g) shows the final PSF for the RAVC2 concept. A perfect cancellation is not obtained because of the spiders (that are not considered in the RAVC2 optimization) and, to a smaller extent, the segments.

\subsection{The Apodized Phase Plate (APP)}

The last concept analyzed here is the Apodized Phase Plate (APP). ${ }^{1,3,23}$ It is a pupil-plane one-stage optical device, consisting of an anti-symmetric phase pattern (see Fig. 4(h)) that redistributes the light in the image plane, creating a dark hole. The APP is placed in PP2, which has a fixed orientation with respect to the input pupil. The vector APP pattern for these simulations is generated using a modified Gerchberg-Saxton algorithm with modified constraints (Keller et el. in prep) where the iterative algorithm was tuned to minimise flux within a 2 to $10 \lambda / D$ region over a $180^{\circ}$ region (see Fig. $4(\mathrm{i})$ ).

\section{RESULTS}

The five possible METIS high-contrast imaging options simulated are here (see Fig. 5): no coronagraph, VC2, RAVC2, VC2+LPM, APP. The simple VC2 does not show much improvement with respect to the noncoronagraphic option, due to the large central obstruction. The VC2 $+\mathbf{L P M}$ and the APP clearly exhibit the characteristic dark holes. Finally the RAVC2 presents the best intensity profile across the FoV. When one AO phase screen is added, the simple VC2 and the APP show very small variations, while the VC2 $+\mathbf{L P M}$ dark hole is partly filled in. The RAVC2 shows the limit of the AO speckles $(1 e-7)$, that cannot be reached by the simple VC2, at least not while the Lyot Stop is not optimized.

The final throughput has also been computed, for a better comparison between the concepts. In particular, two types of throughput have been analyzed: the Encircled Energy (EE) and the Total Energy (TE), both evaluated in the image plane. The former is defined as the ratio between the encircled energy within the FWHM of the off-axis coronagraphic PSF (placed at $10 \lambda / D$ from the optical axis) and the encircled energy of the on-axis non-coronagraphic PSF. In our EE definition, we try to maximize the energy in the FWHM, because it is the size commonly used to detect a companion in the final image. The TE is the integrated energy of the off-axis coronagraphic PSF in the focal plane normalized by the integrated energy of the on-axis non-coronagraphic PSF. The two quantities have also been computed at $0 \lambda / D$ without the VC2 (but with Lyot Stop, LPM or ring apodizer), in order to evaluate the effect of these masks on the final throughput. In particular, the TE of the $\mathrm{VC} 2$ at $0 \lambda / D$ represents the surface area of the Lyot Stop ${ }^{\dagger}$. The TEs of the LPM and the RAVC2 take into account the Lyot Stop (different between the two concepts, bigger for the RAVC, see figure 4(f)) and the

\footnotetext{
${ }^{\dagger}$ Note that the real transmission of the diamond mask is not perfect, producing some loss.
} 


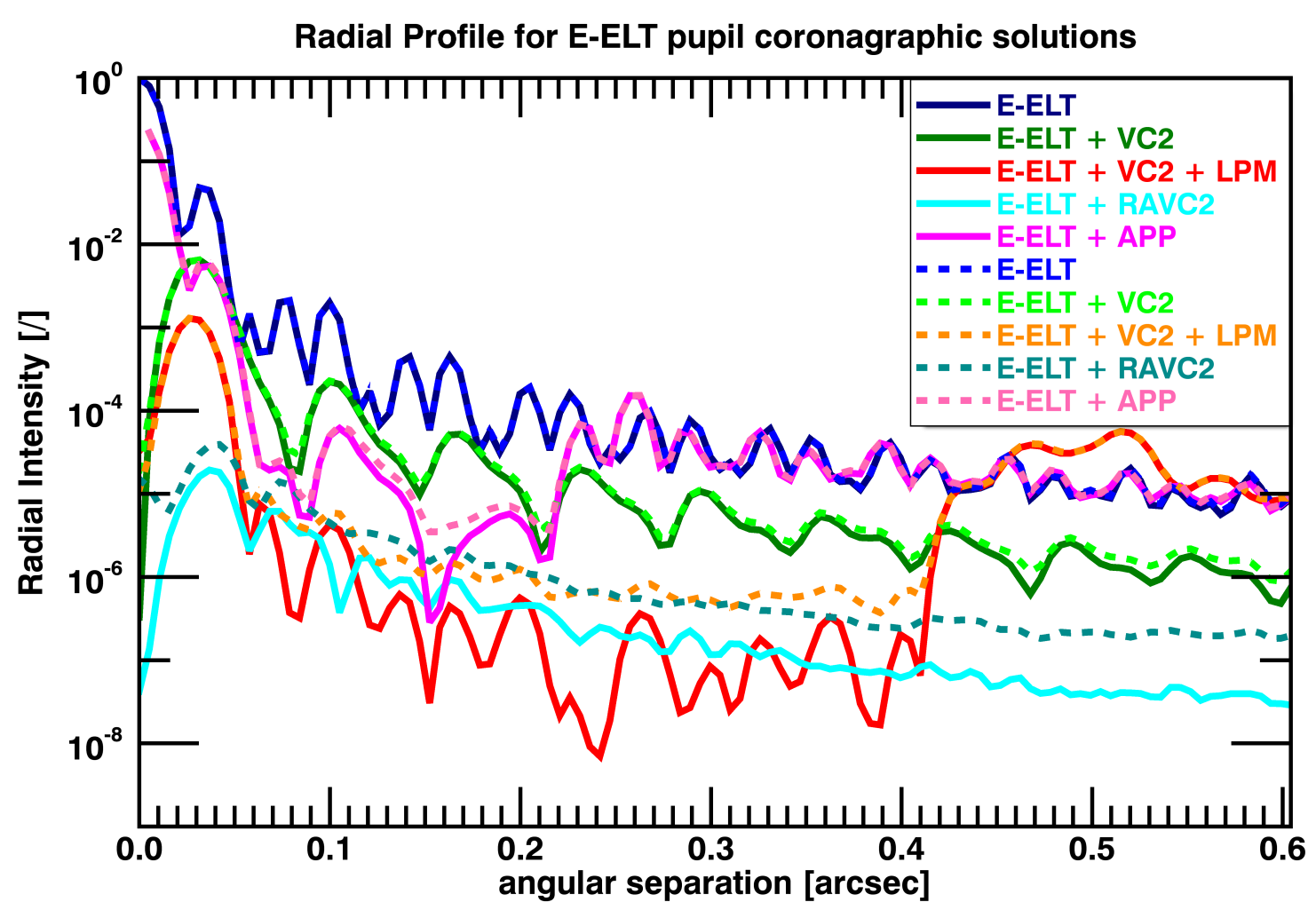

Figure 5: Radial Profile with (dashed lines) and without (full lines) AO screens

trasmittance of the two masks. As expected, the simple VC2 has the highest off-axis EE, while the APP has the highest TE ${ }^{\ddagger}$. The RAVC2 shows the lowest values, while the LPM has been designed to keep a resonable level of throughput $\S$.

\begin{tabular}{|c|c|c|c|c|}
\hline Imaging Concept & $\mathrm{EE}(10 \lambda / D)$ & $\mathrm{TE}(10 \lambda / D)$ & $\mathrm{EE}(0 \lambda / D)$ & $\mathrm{TE}(0 \lambda / D)$ \\
\hline no coro & 1 & 1 & $/$ & $/$ \\
VC2 & 0.81 & 0.88 & 0.95 & 0.97 \\
VC2 + LPM & 0.18 & 0.87 & 0.20 & 0.97 \\
RAVC2 & 0.18 & 0.33 & 0.20 & 0.36 \\
APP & 0.35 & 0.99 & $/$ & $/$ \\
\hline
\end{tabular}

Table 1: Throughput results: the Encircled Energy (EE) and the Total Energy (TE) are both computed as the ratio between the off-axis $(10 \lambda / D$ from the optical axis) coronagraphic PSF and the on-axis coronagraphic PSF. The former considers the energy within the FWHM, the latter the integrated energy over the image plane. The two quantities have also been computed at $0 \lambda / D$ whithout the vortex mask (but with Lyot Stop, LPM or ring apodizer), in order to evaluate the effect of these masks on the final throughput.

\subsection{ADI contrast}

2000 simulated AO phase screens have been used to simulate an ADI observing sequence. They represent 2 sec worth of AO residuals in standard conditions on a bright star. These 2000 phase screens have been used to represent a $1 \mathrm{~h}$ observing sequence on a star transiting at $20^{\circ}$ from zenith (giving a total parallactic angle rotation

\footnotetext{
${ }^{\ddagger}$ Since the APP is theoretically a redistribution of energy, without any loss (in reality some loss is expected), the total energy of the coronagraphic and non-coronagraphic PSF is the same.

${ }^{\S}$ The LPM is a phase only element. This means that the final TE without VC2 should be equal to the surface area of the Lyot Stop.
} 


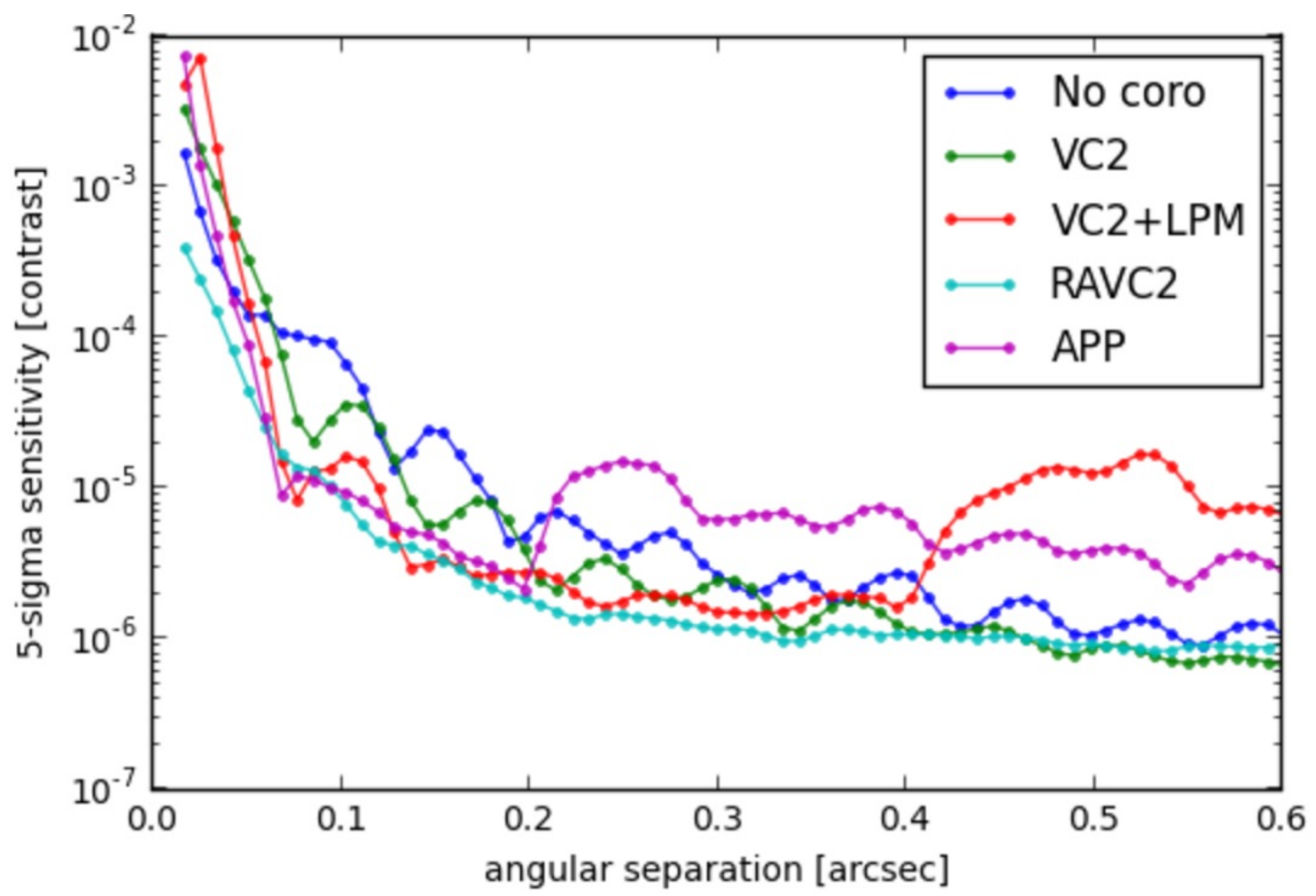

Figure 6: ADI contrast curve

of almost $40^{\circ}$ ). Each phase screen then corresponds to about $1.8 \mathrm{~s}$ of observation. This does not properly capture the time evolution and averaging of speckles. Non-Common Path Aberrations (NCPA) have not been included for now. Firstly, we resample the cubes to the METIS pixel size of 8.6 mas. The inputs from the METIS simulator (courtesy of Jeff Meisner) are then used to convert the PROPER simulations into ADU, assuming a target star of magnitude $\mathrm{L}=5$, and to add the contribution of the thermal background. Using the NACO-Lp filter, an $\mathrm{L}=5$ star is expected to produce $2.40 e 9 \mathrm{ADU} / \mathrm{s}$ on the METIS detector. With its plate scale of $8.6 \mathrm{mas} / \mathrm{pixel}$, the METIS detector also receives $4.32 e 5 \mathrm{ADU} / \mathrm{s}$ / pixel of thermal background emission. We multiply the PROPER simulations by the total stellar flux, and add the background emission multiplied by the transmission of all the pupil stops / apodizers in the beam. Photon noise is then added on top of each frame. Standard PCA-ADI processing ${ }^{24}$ is used to process the cubes, using 10 principal components on 140x140 pixel frames featuring the METIS $8.6 \mathrm{mas} /$ pixel sampling at L band. The noise radial profile is computed in the final image as the standard deviation of the aperture fluxes measured in as many independent resolution elements as possible at a given angular separation from the star. The noise estimation takes into account the small-sample penalty. ${ }^{25}$ For the APP, this estimation is done on the clean half of the FoV. The throughput of the PCA-ADI algorithm is evaluated by injecting fake companions at 10x the noise level using the off-axis PSF as a template, and by comparing their flux in the final image to the injected flux level. The 5-sigma contrast curve is finally obtained by dividing the $5^{*}$ noise radial profile by the algorithm throughput radial profile and by the aperture photometry of the off-axis star (using an aperture diameter $=\lambda / D$ ).

The simple vortex coronagraph (VC2) starts to outperform non-coronagraphic imaging beyond 85mas (i.e., $4 \lambda / D)$, but the gain never exceeds a factor 3 . It could be due to the response of the vortex to low-order aberrations in the presence of a large central obstruction.

The RAVC2 performs better than any other mode. Its strength is to combine a reasonably high transmission with a very low residual starlight level in the focal plane. The gain in sensitivity is up to a factor 10 with respect to non-coronagraphic imaging.

The APP and VC2 $+\mathbf{L P M}$ provide similar performance inside their dark hole, and this performance is on a par with the RAVC2 at the same angular separation. 


\section{CONCLUSIONS}

In this paper, we have presented end-to-end performance simulations of various METIS coronagraphic modes, including focal-plane vortex phase masks and pupil-plane apodizing phase plate. Taking into account the E-ELT pupil architecture, the atmosphere and the AO contributions, our results identify the RAVC2 and the APP as the most promising solutions. It must however be noted that these simulations are still preliminary, as some effects still need to be taken into account. In the future, we plan to (i) use a longer, more representative AO simulation, (ii) include the effect of NCPA, and (iii) include the effect of vibrations.

\section{ACKNOWLEDGMENTS}

The research leading to these results has received funding from the European Research Council under the

European Union's Seventh Framework Programme (ERC Grant Agreement n.337569) and from the French Community of Belgium through an ARC grant for Concerted Research Actions.

\section{REFERENCES}

[1] Kenworthy, M., Codona, J., Hinz, P., Angel, J., Heinze, A., and Sivanandam, S., "First On-Sky HighContrast Imaging with an Apodizing Phase Plate," Astrophysical Journal 660, 762-769 (2007).

[2] Kenworthy, M., Quanz, S., Meyer, M., Kasper, M., Lenzen, R., Codona, J., Girard, J., , and Hinz, P., "An apodizing phase plate coronagraph for VLT/NACO," Proc. SPIE 7735 (2010).

[3] Quanz, S., Meyer, M., Kenworthy, M., Girard, J., Kasper, M., Lagrange, A.-M., Apai, D., Boccaletti, A., Bonnefoy, M., Chauvin, G., Hinz, P., and Lenzen, R., "First Results from Very Large Telescope NACO Apodizing Phase Plate: $4 \mu \mathrm{m}$ Images of the Exoplanet $\beta$ Pictoris b," Astrophysical Journal, Letters $\mathbf{7 2 2}$, L49-L53 (2010).

[4] Kenworthy, M., Hinz, P., Codona, J., Wilson, J., Skrutskie, M., and Solheid, E., "Developing achromatic coronagraphic optics for LMIRCam and the LBT," Proc. SPIE 7734 (2010).

[5] Quanz, S., Amara, A., Meyer, M., Kenworthy, M., Kasper, M., and Girard, J., " A young protoplanet candidate embedded in the circumstellar disk of HD100546," Astrophysical Journal, Letters 766, L49-L53 (2013).

[6] Snik, F., Otten, G., Kenworthy, M., Mawet, D., and Escuti, M., "Combining vector-phase coronagraphy with dual-beam polarimetry," Proc. SPIE 9147 (2014).

[7] Otten, G., Snik, F., Kenworthy, M., and Miskiewicz, M.N.and Escuti, M., "Performance characterization of a broadband vector Apodizing Phase Plate coronagraph," Optics Express 22 (2014).

[8] Otten, G., Snik, F., Kenworthy, M., and Miskiewicz, M.N.and Escuti, M., "The broad-band vector Apodizing Phase Plate (vAPP) coronagraph: NIR liquid crystal performance and future developments," Proc. SPIE 9912 (2016).

[9] Mawet, D., Riaud, P., Absil, O., and Surdej, J., "Annular Groove Phase Mask Coronaghaph," Astrophysical Journal 633, 1191-1200 (2005).

[10] Mawet, D., Riaud, P., Surdej, J., and Baudrand, J., "Subwavelength surface-relief gratings for stellar coronagraphy," Applied Optics 44, 7313-7321 (2005).

[11] Delacroix, C., Forsberg, P., Karlsson, M., Mawet, D., Absil, O., Hanot, C., Surdej, J., and Habraken, S., "Design, manufacturing, and performance analysis of mid-infrared achromatic half-wave plates with diamond subwavelength gratings," Applied Optics 51, 5897 (2012).

[12] Forsberg, P. and Karlsson, M., "High aspect ratio optical gratings in diamond," Diamond and Related Materials 34, 19-24 (2013).

[13] Absil, O., Milli, J., Mawet, D., Lagrange, A.-M., Girard, J., Chauvin, G., Boccaletti, A., Delacroix, C., and Surdej, J., "Searching for companions down to 2 AU from $\beta$ Pictoris using the L'-band AGPM coronagraph on VLT/NACO," Astronomy and Astrophysics 559, L12 (2013). 
[14] Carlomagno, B., Delacroix, C., Absil, O., Forsberg, P., Habraken, S., Jolvet, A., Karlsson, M., Mawet, D., Piron, P., Surdej, J., , and Vargas Catalan, E., "Mid-IR AGPMs for ELT applications," Proc. SPIE 9147 (2014).

[15] Brandl, B., Lenzen, R., Pantin, E., Glasse, A., Blommaert, J., Venema, L., Molster, F., Siebenmorgen, R., Boehnhardt, H., van Dishoeck, E., van der Werf, P., Henning, T., Brandner, W., Lagage, P.-O., Moore, T., Baes, M., C., W., Wright, C., Kaufl, H., Kendrew, S., Stuik, R., , and Jolissant, L., "METIS- the Mid-infrared E-ELT Imager and Spectrograph," Proc. SPIE 7014 (2008).

[16] Kenworthy, M., "High-contrast imaging with METIS," Proc. SPIE 9908-378 (2016).

[17] Feldt, M., "Sensing wavefronts on resolved sources with pyramids on ELTs," Proc. SPIE 9909 (2016).

[18] Stuik, R., "Designing the METIS SCAO and LTAO systems," Proc. SPIE 9909-11 (2016).

[19] Krist, J., PROPER. An Optical Propagation Library for IDL (2008).

[20] Ruane, G., Huby, E., Absil, O., Mawet, D., Delacroix, C., B., C., and G.A., S., "Lyot-plane phase masks for improved high-contrast imaging with a vortex coronagraph," Astronomy and Astrophysics $\mathbf{5 8 3}$ (2015).

[21] Ruane, G., Absil, O., Huby, E., Mawet, D., Delacroix, C., Carlomagno, B., Piron, P., and Swartzlander, G., "Optimized focal and pupil plane masks for vortex coronagraphs on telescopes with obstructed apertures," Proc. SPIE 9605 (2015).

[22] Mawet, D., Pueyo, L., Carlotti, A., Mennesson, B., Serabyn, E., and J.K., W., "Ring-Apodized Vortex Coronagraphs for Obscured Telescopes. I. Transmissive Ring Apodizers," Astrophysical Journal Supplement 209 (2013).

[23] Codona, J., Kenworthy, M., Hinz, P., Angel, J., and Woolf, N., "A high-contrast coronagraph for the MMT using phase apodization: design and observation at 5 microns and $2 \lambda / D$ radius," Proc. SPIE 6269 (2006).

[24] Gomez Gonzalez, C. A., Wertz, O., Christiaens, V., Absil, O., and Mawet, D., "VIP: Vortex Image Processing pipeline for high-contrast direct imaging of exoplanets." Astrophysics Source Code Library (Mar. 2016).

[25] Mawet, D., Milli, J., Wahhaj, Z., Pelat, D., Absil, O., Delacroix, C., Boccaletti, A., Kenworthy, M., Marois, C., Mennesson, B., and Pueyo, L., "Fundamental Limitations of High Contrast Imaging Set by Small Sample Statistics," Astrophysical Journal 792 (2014). 